Please do not remove this page

RMIT

UNIVERSITY

\title{
Elemental analogues of graphene: Silicene, germanene, stanene, and phosphorene
}

Balendhran, Sivacarendran; Walia, Sumeet; Nili Ahmadabadi, Hussein; Sriram, Sharath; Bhaskaran, Madhu

https://researchrepository.rmit.edu.au/esploro/outputs/9921862149701341/filesAndLinks?institution=61RMIT_INST\&index=null

Balendhran, S., Walia, S., Nili Ahmadabadi, H., Sriram, S., \& Bhaskaran, M. (2015). Elemental analogues of graphene: Silicene, germanene, stanene, and phosphorene. Small, 11(6), 640-652.

https://doi.org/10.1002/smll.201402041

Document Version: Accepted Manuscript

Published Version: https://doi.org/10.1002/smll.201402041

Repository homepage: https://researchrepository.rmit.edu.au

(c) 2014 Wiley-VCH Verlag GmbH \& Co. KGaA, Weinheim

Downloaded On 2023/04/26 17:52:58 +1000 
Thank you for downloading this document from the RMIT Research Repository.

The RMIT Research Repository is an open access database showcasing the research outputs of RMIT University researchers.

RMIT Research Repository: http://researchbank.rmit.edu.au/

\section{Citation:}

Balendhran, S, Walia, S, Nili Ahmadabadi, H, Sriram, S and Bhaskaran, M 2015, 'Elemental analogues of graphene: Silicene, germanene, stanene, and phosphorene', Small, vol. 11, no. 6, pp. 640-652.

See this record in the RMIT Research Repository at:

https://researchbank.rmit.edu.au/view/rmit:29862

Version: Accepted Manuscript

Copyright Statement:

(C) 2014 Wiley-VCH Verlag GmbH \& Co. KGaA, Weinheim

\section{Link to Published Version:}

http://dx.doi.org/10.1002/smll.201402041 
DOI: $10.1002 /$ ((please add manuscript number))

Article type: Review Article

\section{Elemental Analogues of Graphene: Silicene, Germanene, Stanene and Phosphorene}

Sivacarendran Balendhran, * Sumeet Walia, Hussein Nili, Sharath Sriram, and Madhu Bhaskaran.*

Dr. S. Balendhran, Dr. S. Walia, Mr. H. Nili, Dr. S. Sriram, and Dr. M. Bhaskaran. Functional Materials and Microsystems Research Group, RMIT University, Melbourne, Victoria 3001, Australia

E-mail: shiva.balendhnran@rmit.edu.au,madhu.bhaskaran@rmit.edu.au

Keywords: silicene, graphene analogues, two-dimensional, germanene, phosphorene

Abstract: The fascinating electronic and optoelectronic properties of free-standing graphene has led to the exploration of alternative two-dimensional materials that can be easily integrated with current generation of electronic technologies. In contrast to 2D oxide and dichalcogenides, elemental 2D analogues of graphene, which include monolayer silicon (silicene), are fast emerging as promising alternatives, with predictions of high degree of integration with existing technologies. This article reviews this emerging class of $2 \mathrm{D}$ elemental materials - silicene, germanene, stanene, and phosphorene - with emphasis on fundamental properties and synthesis techniques. The need for further investigations to establish controlled synthesis techniques and the viability of such elemental 2D materials is highlighted. Future prospects harnessing the ability to manipulate the electronic structure of these materials for nano- and opto-electronic applications are identified. 


\section{Introduction}

The prospect of exploiting the fascinating electrodynamics of the quantum realm using twodimensional (2D) materials, has initiated a paradigm shift towards the exploration of sophisticated 2D systems. The most striking has been the emergence of graphene, the hexagonal planar allotrope of carbon which has driven the pursuit for high speed nanoelectronics. Its free standing $2 \mathrm{D}$ nature, gives rise to some of its exotic and favorable aspects that are lacking in its bulk counterpart (graphite). This includes extraordinarily enhanced electronic properties that arise due to the quantum confinement of the carriers along the plane and carriers acting as relativistic massless Dirac femions, where they can travel exceptional distances without scattering. ${ }^{[1-2]}$ The presence of such Dirac fermions also ensures a minimum (quantum) electrical conductivity, even at a free carrier concentration of zero. ${ }^{[2]}$ Such exceptional aspects of graphene are envisioned to be the foundation of the future generation of nanoelectronics. However, the semi-metallic and chemically inert nature of graphene proves to be a hindrance in utilizing its full potential. Additionally, it lacks the characteristic digital switching transitions that have underpinned the success of silicon based electronic technologies. As such, the discovery of graphene has initiated a runaway effect in the pursuit of alternative versatile 2D materials for electronic, optoelectronic, catalysis energy storage and sensing applications. ${ }^{[3-4]}$

The quest for graphene analogues has resulted in the exploration of a variety of materials ranging from transition metal chalcogenides $\left.\left(\mathrm{MoS}_{2}, \mathrm{MoO}_{3}, \mathrm{MoSe}_{2}, \mathrm{WS}_{2}, \mathrm{WSe}\right)_{2}\right)$, hexagonal boron nitride $(\mathrm{hBN})$, to other elemental p-block materials $(\mathrm{Si}, \mathrm{Ge}) .{ }^{[3-10]}$ While there have been significant advancements in knowledge about such 2D semiconducting materials, they still have not been able to outperform graphene in terms of ballistic transport properties. This can be attributed to the density of electronic states and the quasi 2D nature of these materials at an atomic scale, in contrast to the elemental configuration of graphene. 
In recent years, elemental sheets of silicon and germanium (silicene and germanene respectively) have been emerging as strong contenders in the realm of $2 \mathrm{D}$ materials. ${ }^{[5,7]}$ There have been several theoretical studies assessing their fundamental properties while experimental analyses are just in their infancy, as practical synthesis methods are being explored to establish well defined fabrication techniques and parameters. Studies predict that such elemental sheets may also possess Dirac fermions similar to graphene and much simpler techniques may become available for their bandgap engineering. Although semi-metallic, the main hurdle experienced in realizing silicene and germanene is that unlike graphene, they do not form a van der Waals layered structures in their bulk phase. Hence, they do not exist as free standing sheets but synthesized as adlayer structures on ordered substrates. ${ }^{[11]}$ Despite this fact, the exceptional findings through theoretical and preliminary experimental analyses, along with its compatibility to the current silicon based electronics, continues to inspire the exploration of 2D silicene and other Group IV elemental materials (germanene, stanene). Very recently, phosphorene (the fundamental 2D structure that composes black phosphorus crystals) which exists as a freestanding 2D nanosheet, has also been introduced to the family of elemental materials, as an excellent semiconductor with graphene like properties. However, a lot remains to be explored before these materials can be established as viable alternatives for the next generation of electronic applications. ${ }^{[12-13]}$

As such, this review aims to cater for the increasing demand in knowledge that has risen due to the pursuit of elemental 2D materials for electronic applications. Comprehensive reviews analyzing a wide spectrum of $2 \mathrm{D}$ materials are available elsewhere. ${ }^{[3-5,14]}$ Reviews detailing the fundamental properties of silicene and related theoretical simulations are also available. ${ }^{[7,}$ ${ }^{15]}$ In this review, we focus on delivering a comprehensive analysis of the fundamental properties, synthesis approaches and the future prospects of silicene, germanene, stanene and phosphorene. 


\section{Fundamental Properties}

This section will cover the literature on the fundamental properties of graphene analogous elemental sheets, inclusive of both theoretical and experimental knowledge. However, most of the properties discussed herein are based on theoretical analyses as the lack of an established facile synthesis technique has proved to be a hindrance to experimental studies. Although there is a lack of knowledge on the fundamental properties of germanene and stanene, the striking similarity in their crystal and electronic structures, enable the properties of germanene and stanene to be extrapolated through comparisons with graphene and silicene.

\subsection{Crystal Structure}

This section provides an insight into the fundamental crystal arrangements of elemental structures. The crystal structures are mainly categorized as elemental, decorated and composite structures.

\subsubsection{Elemental Structures}

The atomic arrangements of planar Group IV elements (C, Si, Ge, and $\mathrm{Sn}$ ) follow the hexagonal honeycomb structure of graphene. In their stable state, these elements form a buckled hexagonal structure, unlike graphene, which forms a perfect planar layer with all carbon atoms bonded in a single plane. ${ }^{[16-17]}$ This is related to the nature of graphene possessing a $s p^{2}$ hybridization, whereas $\mathrm{Si}, \mathrm{Ge}$, and $\mathrm{Sn}$ exhibit the $s p^{2}-s p^{3}$ hybridization due to their preferential state of $s p^{3}$ bonding. Figure 1a illustrates this hexagonal structure and the buckled nature, where some atoms of the unit cell are in a lower atomic plane. Each $\mathrm{X}$ atom $(\mathrm{X}=\mathrm{Si}, \mathrm{Ge}$ and $\mathrm{Sn})$ is covalently bonded with three other $\mathrm{X}$ atoms, resulting in a simple hexagonal unit cell. The buckling occurs at various points in the unit cell, resulting in different types of lattice arrangements such as chair-, boat- and washboard-like structures (Figure 1b). ${ }^{[16]}$ Density functional studies reveal that the boat- and washboard-like arrangements are unstable and they converge into the flat structure, while the chair-like structure remains more stable than the flat structure. ${ }^{[16]}$ The vertical distance separating the 
two atomic planes in these structures is known as the buckling parameter $(\delta)$. Variation in the buckling parameter gives rise to changes in electronic properties, governed by the density of electronic states. Table 1 presents the lattice and buckling parameters (relatively stable lowbuckled structures) for selected elemental sheets.
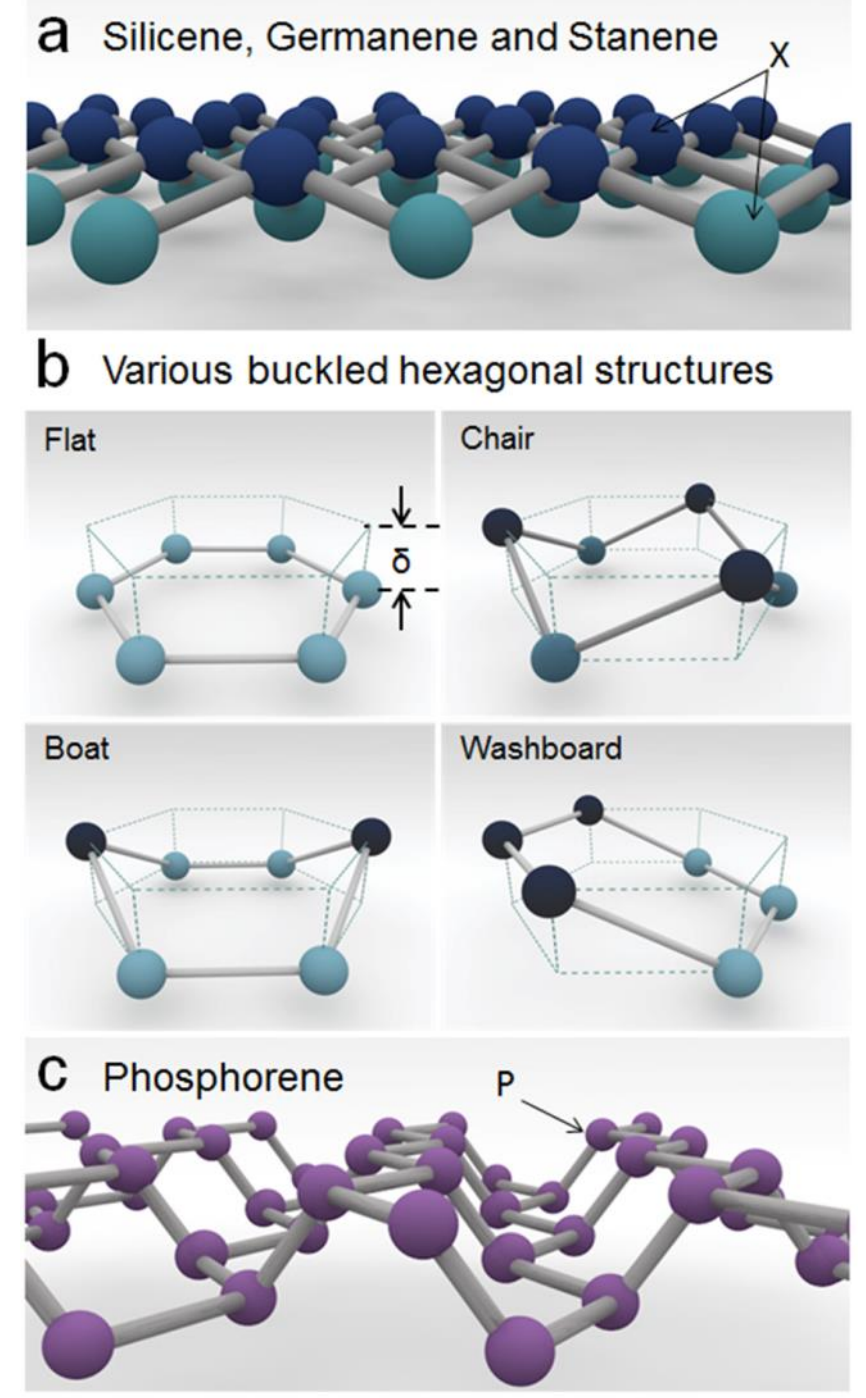

Figure 1. (a) Buckled hexagonal crystal structure of $2 \mathrm{D}$ elemental sheets $(X=\mathrm{Si}, \mathrm{Ge}$, and Sn). Darker shaded atoms are at a slightly higher horizontal plane than the lighter shaded atoms. (b) Various hexagonal buckled structures of $X$. The buckling parameter $\delta$ is the vertical distance between the two planes of $\mathrm{X}$ atoms (c) Puckered hexagonal crystal structure of phosphorene. 
Table 1. Structural and electronic parameters of elemental structures. Electronic quantities for Group IV elements are derived from hybrid exchange-correlation functional HSE06 calculations with the inclusion of spin orbit coupling. ${ }^{[18]}$

\begin{tabular}{|c|c|c|c|c|c|}
\hline & C & Si & Ge & Sn & $\mathbf{P}$ \\
\hline Lattice constant $a(\mathrm{~nm})$ & 0.2468 & 0.3868 & 0.4060 & 0.4673 & $0.453^{[13]}$ \\
\hline Bond length $d(\mathrm{~nm})$ & 0.1425 & 0.2233 & 0.2344 & 0.2698 & $0.336^{[13]}$ \\
\hline Buckling parameter $\delta(\mathrm{nm})$ & 0 & 0.045 & 0.069 & 0.085 & $0.500^{[12}$ \\
\hline Effective electron mass $m^{*}\left(m_{0}\right)$ & 0 & 0.001 & 0.007 & 0.029 & - \\
\hline Fermi velocity of carriers $V_{\mathrm{F}}\left(10^{6} \mathrm{~ms}^{-1}\right)$ & 1.01 & 0.65 & 0.62 & 0.55 & - \\
\hline Energy gap $E_{\mathrm{g}}(\mathrm{meV})$ & 0.02 & 1.9 & 33 & 101 & $1000^{[13}$ \\
\hline In-plane stiffness $C\left(\mathrm{Jm}^{-2}\right)$ Pristine ${ }^{[19]}$ & - & 100 & 53 & 38 & - \\
\hline Ni-doped ${ }^{[19]}$ & - & 138 & 72 & 63 & - \\
\hline
\end{tabular}

Phosphorene is the fundamental atomic layer that composes the naturally existing black phosphorous, which is the known thermodynamically stable form of phosphorous. ${ }^{[12-13]}$ Similar to graphite, black phosphorous is made of vertically stacked layers of phosphorene held together by weak van der Waals forces. ${ }^{[20]}$ Unlike graphene and other Group IV elemental sheets, phosphorene possesses a unique, vertically skewed/wrinkled hexagonal structure (Figure 1c), where each phosphorus $(\mathrm{P})$ atom is covalently bonded to three other $\mathrm{P}$ atoms, resembling a steep washboard-like structure. ${ }^{[12]}$

\subsubsection{Decorated Structures}

Decorated structures involve a foreign element covalently bonded to each atom of the $2 \mathrm{D}$ sheets in the cross planar direction. Considering their valance band, the decorated structures are more stable in comparison to their elemental counterparts and hence they can be separated as free standing sheets. Theoretical studies based on hydrogenated and fluorinated elementals sheets further reveal the stability of the decorated structures over their elemental counterparts. ${ }^{[21]}$ The common bonding preferences for various buckled structures of decorated Group IV elements are illustrated in Figure 2a. ${ }^{[22]}$ Typical formation of decorated, flat (nonbuckled) structure is known to exhibit a table like crystal phase, where the decorating elements lay in a single plane. This type of structure is also observed in substrate assisted growth of elemental 2D sheets, where instead of the decorating element, the sheets covalently bond with the substrate. Stable chair-like buckled crystals form a symmetrical 1 up, 1 down 
structure (Figure 2a), where the 2D sheets are sandwiched between the decorating elements. ${ }^{[22]}$ Such structures allow free standing sheets of decorated crystals to be realized as in the case of hydrogen terminated silicene and germanene (known as silicane and germanane respectively). ${ }^{[23-24]}$ Although unstable, boat- and washboard-like buckled structures are predicted to form a slightly distorted arrangement (2 up, 2 down and 3 up, 3 down respectively). ${ }^{[22]}$ Table 2 presents the structural and buckling parameters of hydrogen decorated Group IV elemental sheets. Sahin et al. have conducted detailed theoretical studies on the adsorption of a range of alkali, alkaline-earth, and $3 \mathrm{~d}$ transition metal atoms on silicene. ${ }^{[25]}$ They identified various adsorption sites in silicene favored by different adatoms and the consequent changes in the electronic properties of silicene, which can be utilized in applications. ${ }^{[25]}$

Figure 2. (a) Various geometrical configurations of decorated, $2 \mathrm{D}$ elemental sheets. In this case, the elemental structures are decorated with hydrogen (X denotes $\mathrm{Si}, \mathrm{Ge}$ and $\mathrm{Sn})$. The typical decoration sites desired by hydrogen depending on the lattice structure are presented. (b) Typical configurations of composite structures for silicene, germanene and stanene.

Table 2. Structural and electronic parameters of hydrogenated elemental structures calculated using local density approximation excluding spin-orbit coupling. ${ }^{[26]}$

\begin{tabular}{lllll}
\hline & $\mathrm{CH}$ & $\mathrm{SiH}$ & $\mathrm{GeH}$ & $\mathrm{SnH}^{[21]}$ \\
\hline Lattice constant $a(\mathrm{~nm})$ & 0.2514 & 0.3820 & $0.4091^{[21]}$ & 0.4719 \\
Metal-metal length $d(\mathrm{~nm})$ & 0.1520 & 0.2319 & 0.2338 & 0.2846 \\
Metal-hydrogen length $(\mathrm{nm})$ & 0.1084 & 0.1502 & 0.1530 & 0.1738 \\
Buckling parameter $\delta(\mathrm{nm})$ & 0.045 & 0.072 & 0.069 & 0.082 \\
Fermi velocity of carriers $V_{\mathrm{F}}\left(10^{6} \mathrm{~ms}^{-1}\right)$ & 0.63 & 0.51 & 0.38 & - \\
Energy gap $E_{\mathrm{g}}(\mathrm{eV})$ & $4.9^{\mathrm{b})}, 5.4^{\text {c) }[27]}$ & $2.9^{\mathrm{b})}, 4.0^{\text {c) }[28]}$ & $2.9^{\mathrm{b})}, 3^{\text {c) }[28]}$ & - \\
\hline
\end{tabular}

b) and c): boat- and chair-like crystal structures respectively.

\subsubsection{Composite Structures}

The classic composite structures of Group IV elements form YX and $\mathrm{YX}_{3}$ structures (where $\mathrm{Y}=\mathrm{B}, \mathrm{C}, \mathrm{N}, \mathrm{Al}, \mathrm{P}, \mathrm{Ni}, \mathrm{Fe}$, and Co). ${ }^{[16,19]}$ Typical atomic arrangements of $\mathrm{YX}$ and $\mathrm{YX}_{3}$ are depicted in Figure 2b. YX composites form various buckled structures depending on the dopant as depicted by the unit cells in Figure $1 b .{ }^{[16,19]}$ However, through first principle studies, the $\mathrm{YX}_{3}$ structures are expected to form flat graphene like structures with $\mathrm{B}, \mathrm{Al}$, and $\mathrm{C}$ while chair-like buckled structures are seen with $\mathrm{N}$ and $\mathrm{P} \cdot{ }^{[16]}$ Such a preference is attributed to the 
number of valance electrons of the dopant with respect to the $\mathrm{X}$ atoms. As $\mathrm{N}$ and $\mathrm{P}$ atoms contain more valance electrons than $\mathrm{X}$, the composite structure is highly $n$-doped, where these electrons cause extra compression strain resulting in a buckled structure. ${ }^{[16,29]}$ This behavior can also explain the buckled structural nature of transition metal composites.

\subsection{Electronic Properties}

\subsubsection{Electronic Band Structure}

Similar to graphene, silicene is predicted to be a gapless semimetal with $s p^{2}$ hybridization. ${ }^{[30-}$ ${ }^{31]}$ However this allotrope is considered unstable and silicene is commonly observed in a metastable buckled phase due to the preferential $s p^{3}$ hybridization of Si atoms (also observed in the case of Ge and $\mathrm{Sn}$ ). Linear energy-momentum dispersion coupled with a large Fermi velocity $\left(1.2 \times 10^{6} \mathrm{~ms}^{-1}\right)$, derived from quasi particle interference (QPI) patterns, reveal the existence of the Dirac cone in the electronic band structure of low-buckled silicene. ${ }^{[32-33]}$ Experimental evidences of silicene overlaid on Ag point to Fermi velocities comparable to that of graphene and the nature of the quasi particles in silicene behaving as massless Dirac fermions. ${ }^{[32,34]}$ However, in such cases, any underlying substrate induced effects should be isolated in order to determine their intrinsic properties. ${ }^{[35-36]}$ Germanene and stanene, apart from possessing a slightly metallic band alignment, are predicted to possess massless Dirac fermions and exhibit other electronic properties, similar to that of silicene. ${ }^{[30-31]}$

\subsubsection{Quantum Spin Hall (QSH) Effect}

QSH effect is caused by an opening of a bandgap at Dirac point induced by the spin-orbit coupling, which facilitates the $2 \mathrm{D}$ material transition from semi-metallic to a QSH insulator. ${ }^{[37]}$ In graphene, the proposed QSH effect is weak and can only occur at extremely low inaccessible temperatures. ${ }^{[37-38]}$ So far this QSH effect has only been demonstrated in HgTe quantum well structures at temperature below $10 \mathrm{~K} \cdot{ }^{[39-40]}$ However, density functional theory (DFT) studies show that silicene has a larger spin-orbit coupling strength (in comparison to that of graphene) which may lead to a larger energy gap at the Dirac point 
$(1.55 \mathrm{meV})$ resulting in a significant QSH effect, in an accessible temperature regime. ${ }^{[38]}$ Germanene is also expected to produce an energy gap of $\sim 23.9 \mathrm{meV}$ induced by spin-orbit coupling at much higher temperatures of $277 \mathrm{~K},{ }^{[38]}$ while stanene films are predicted to produce the largest gap $(\sim 300 \mathrm{meV})$ amongst the Group IV honeycomb structures. ${ }^{[17,33,41]}$ Such aspects of the elemental structures create opportunities for the realization of practical spintronics applications. ${ }^{[17]}$

Matthes et al. has conducted theoretical analyses of spin orbit coupling interactions comparing all Group IV elemental sheets. ${ }^{[18]}$ Electronic parameters obtained from the studies are presented in Table 1. The energy gaps introduced by the spin orbit interactions were observed to increase in magnitude with increasing atomic number. Even though such gaps lead to Dirac particles with finite mass, their influence on the Fermi velocity was far greater than the particle mass. ${ }^{[18]}$ Figure 3 illustrates the band structures obtained from the theoretical analyses with the inclusion of spin-orbit interactions. Band structures denoted by the black lines without spin orbit interactions (Figure 3 inset) portray the graphene like electronic signature of the other Group IV elemental sheets.
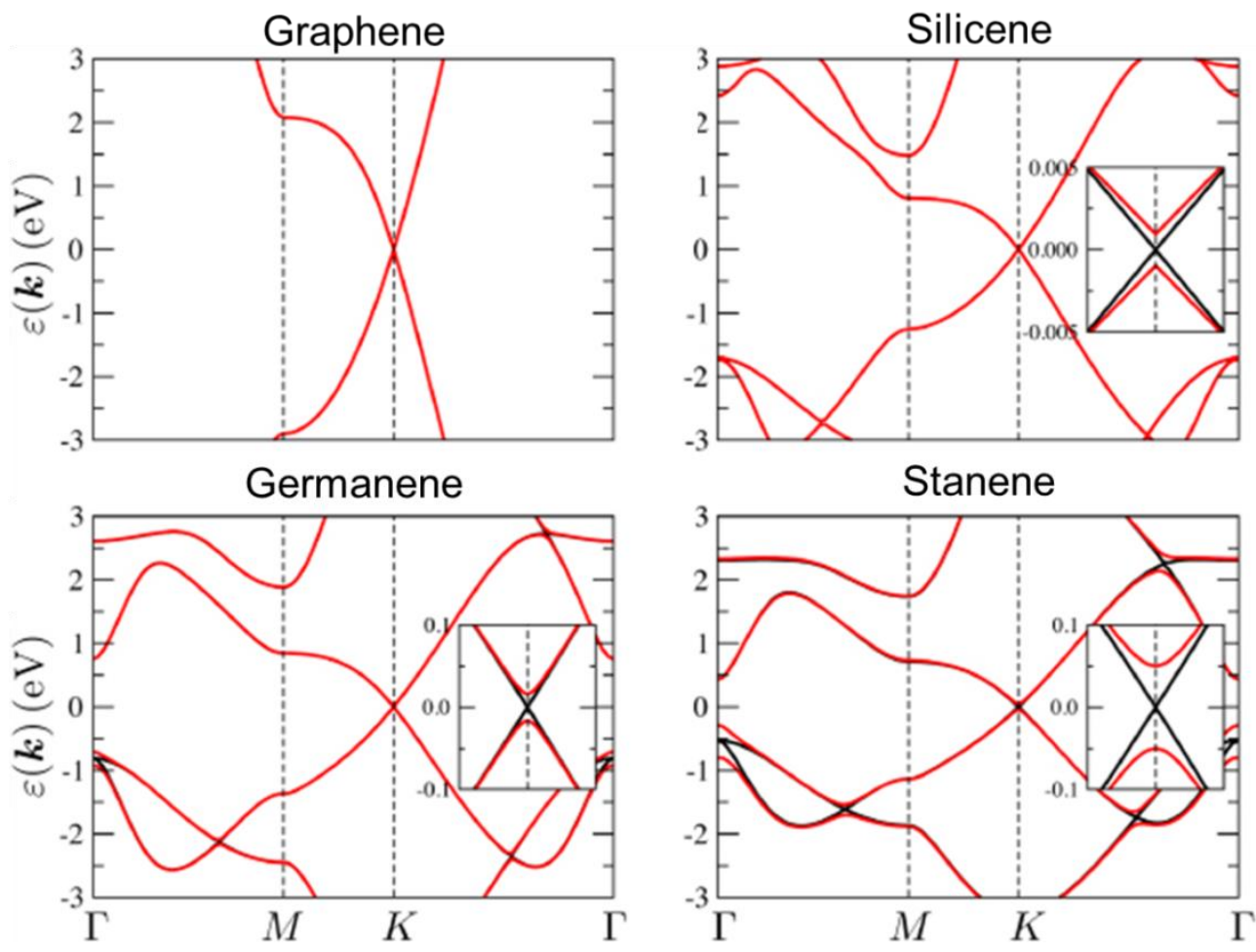
Figure 3. Quasi-particle band structures of Group IV elemental structures along highsymmetry lines of the 2D hexagonal Brillouin zone, using the exchange-correlation functional HSE06 including spin-orbit interactions. The inset shows a magnification of the electronic structure around the Dirac point with the solid black lines indicating the band structure obtained without spin-orbit coupling. Reproduced with permission.$^{[18]}$ Copyright 2013, IOP Publishing.

\subsubsection{Tunability of Electronic Properties}

van der Waals interactions between the substrates and the 2D materials influence their electronic structures. Such effects can be exploited for the band structural engineering of silicene and germanene by overlaying them on various substrates (including graphene). ${ }^{[42-43]}$ Furthermore, theoretical calculations predict the possibility of introducing a tunable bandgap via the application of an electric field, ${ }^{[44-47]}$ functionalization techniques such as hydrogenation/fluorination, ${ }^{[26,28,48-49]}$ and surface adsorption of dopants. ${ }^{[25,50]}$ Hydrogen decorated silicane and germenane are free standing semiconductors that exist in a bulk layered crystal form.

Table 2 presents the electronic parameters of graphane, silicane and germanane. Silicane is predicted to have an indirect gap $(3.8-4.0 \mathrm{eV})$ for chair-like structure and a direct gap (2.9$3.3 \mathrm{eV}$ ) for boat-like structure, while germanane is expected to possess a direct gap for both chair-like $(3.5-3.6 \mathrm{eV})$ and boat-like $(2.9 \mathrm{eV})$ structures. ${ }^{[28]}$ However, these structures are considered wide bandgap structures in comparison to their intrinsic bulk counterparts silicon and germanium (where bandgap values are $<1.2 \mathrm{eV}$ ). The possibility of the Dirac point being preserved at saturated levels of hydrogenation provides an opportunity for silicane and germanane to be adapted in to a wide range of optoelectronic applications.

Wang et al. have reported theoretical studies of oxidized silicene and germanene ( $\mathrm{SiO}$ and GeO) structures. ${ }^{[51]}$ Such oxidized structures are predicted to be direct gap semiconductors. The charge carrier mobilities of $\mathrm{SiO}$ and $\mathrm{GeO}$ are calculated to be in the order of $10^{4} \mathrm{~cm}^{2} \mathrm{~V}^{-1} \mathrm{~s}^{-}$ ${ }^{1}$ and are in a comparable range to that of graphene. Experimental studies confirm the strong resistance of silicene towards oxidation while defect engineering is known to facilitate it. ${ }^{[52]}$ 
Utilizing such characteristics opens a pathway to pattern tailored silicene based topological structures catering to various electronic applications. Composite structures of silicene $\left(\mathrm{SiC}_{3}\right)$ have also been reported to exhibit peculiar band alignments from direct gap semiconducting to gapless semimetallic, depending on the atomic arrangements. ${ }^{[53]}$ Such aspects of tunability in the electronic structures can be harnessed to realize high performance optoelectronic and photovoltaic applications.

\subsubsection{Phosphorene}

Although initial studies on black phosphorus date back to 1950's, only recently phosphorene has attracted interest as an elemental 2D material. Due to its layered nature and high electrical conductivity black phosphorus and its composites were of main interest in battery electrode applications. ${ }^{[54-56]}$ Monolayer phosphorene, in contrast to Group IV elemental sheets, is a semiconductor with a predicted direct bandgap of $1.0 \mathrm{eV},{ }^{[13]}$ which exhibits exceptional layer dependent photoluminescence. ${ }^{[57]}$ For bulk black phosphorus, interlayer van der Waals interactions reduce the bandgap for each additional layer ( $\operatorname{similar}$ to that of $\mathrm{MoS}_{2}$ ) and above 5 layers it converges at $\sim 0.3 \mathrm{eV} \cdot{ }^{[12-13,58-60]}$ Such layer dependent bandgap transitions have also been proved via experimental studies on few layer phosphorene. ${ }^{[61]}$ Theoretical studies also report hydrogen passivation as a favorable route to produce stable direct gap phosphorene nanoribbons, where edge termination (controlled by temperature and partial pressure) determines the degree of tunability. ${ }^{[62]}$ Furthermore, from first principle studies, strain engineering has been predicted to effectively tune the electronic properties of phosphorene. ${ }^{[63-}$ ${ }^{66]}$ ] Recent reports reveal that strain can be utilized as an efficient tool to control the effective electron mass and the direction of electron mobility, ${ }^{[63]}$ as well as induce direct to indirect transitions in the bandgap. ${ }^{[65,67]}$ Such aspects render phosphorene as an excellent candidate for flexible displays, optoelectronic and nanoelectromechanical applications.

Very recently, several reports on few layer black phosphorus utilized in field effect transistors (FETs) project phosphorene as a highly competitive contender in the field of 2D 
nanoelectronics, owing to its desirable electronic and structural characteristics. ${ }^{[12-13,68-71]}$ While the initial reports demonstrate experimental (room temperature) carrier mobilities of up to $1000 \mathrm{~cm}^{2} \mathrm{~V}^{-1} \mathrm{~s}^{-1}$ in few layer quasi $2 \mathrm{D}$ phosphorene, ${ }^{[12]}$ much of the electronic properties of mono-layer phosphorene are yet to be explored. Few layered black phosphorus has also been successfully integrated into photo transistors with responses comparable to $2 \mathrm{D} \mathrm{MoS}_{2},{ }^{[72-73]}$ while its hetero structures with boron nitride and $\mathrm{MoS}_{2}$ (p-n junction diodes) exhibit a photovoltaic effect. ${ }^{[74-76]}$ Furthermore, theoretical studies on the electronic states of phosphorene also indicate a high binding selectivity towards nitrogen based gas molecules portraying its viability for toxic gas sensing applications. ${ }^{[77]}$ The narrow band gap nature of phosphorene and predicted tunability of its properties, makes it an ideal candidate for nanoelectronic, optoelectronic and photovoltaic applications. We believe that this material demands rapid focus to fully unravel its true potential.

\subsection{Mechanical Properties}

The mechanical characteristics of any two-dimensional materials are of utmost importance for assessing their viability for nanoelectromechanical systems (NEMS). In this context, there are a few theoretical studies that have been conducted for the materials covered in this review. Manjananth et al. have carried out theoretical studies on the in-plane stiffness of Group IV elemental sheets for both doped (transition metals such as $\mathrm{Fe}, \mathrm{Co}$ and $\mathrm{Ni}$ ) and pristine structures in various geometries. ${ }^{[19]}$ In all cases, the general the stiffness is observed to reduce with increasing atomic weights (from $\mathrm{Si}$ to $\mathrm{Sn}$ ). This observation is related to the tendency of metallic bonding with increasing atomic weight that is evidenced by the increasing bond length. ${ }^{[19]}$ In both cases of doped and pristine structures, flat planar geometry is observed to have higher stiffness values than that of buckled structures (Table 1). Such a phenomenon has been experimentally shown to occur in graphene, where it exhibits an exceptional mechanical strength. This is expected as the flat geometry possesses much stronger $s p^{2}$ hybridization than that of $s p^{2}-s p^{3}$ hybridization seen in the buckled geometry. ${ }^{[19]}$ Although this makes planar 
sheets ( $\mathrm{Si}, \mathrm{Ge}$ and $\mathrm{Sn}$ ) mechanically stronger than their buckled counterparts, their existence is not energetically favorable and hence they are only observed to occur in a buckled geometry.

Doping the elemental structures with transition metal atoms has been observed to increase the in-plane stiffness in comparison to that of pristine sheets (Table 1). ${ }^{[19]} \mathrm{Ni}$ doping increases the stability of the structures, while reinforcing the in-plane mechanical strength (stiffness values in Table 1). Such behavior is also predicted in the case of doping with other transition metals such as Fe and Co. ${ }^{[19]}$ Based on theoretical studies, Wang et al. have reported strain induced self-doping in silicene and germanene, where large compressive strains are predicted to induce n-type doping, while the tensile strains are predicted to induce p-type doping. ${ }^{[78]}$ Such fascinating aspects of silicene and germanene can allow facile control in engineering their properties in comparison to graphene.

The superior mechanical flexibility of silicene in comparison to that of graphene, has been reported by $\mathrm{Hu}$ et al. ${ }^{[79]}$ The Poisson's ratio (PR), which relates the transverse compression of a material caused by an axial strain, is observed to rapidly increase for silicene, whereas for graphene a steady decline is seen (Figure 4a and b). Such mechanical flexibility in silicene is attributed to the changing bond angle rather than the bond length, while for graphene, the $\mathrm{C}-\mathrm{C}$ bond length is observed to increase with axial strain. Such a unique property in silicene can allow conformity with flexible platforms making it highly adaptable for flexible nanoelectronics. 

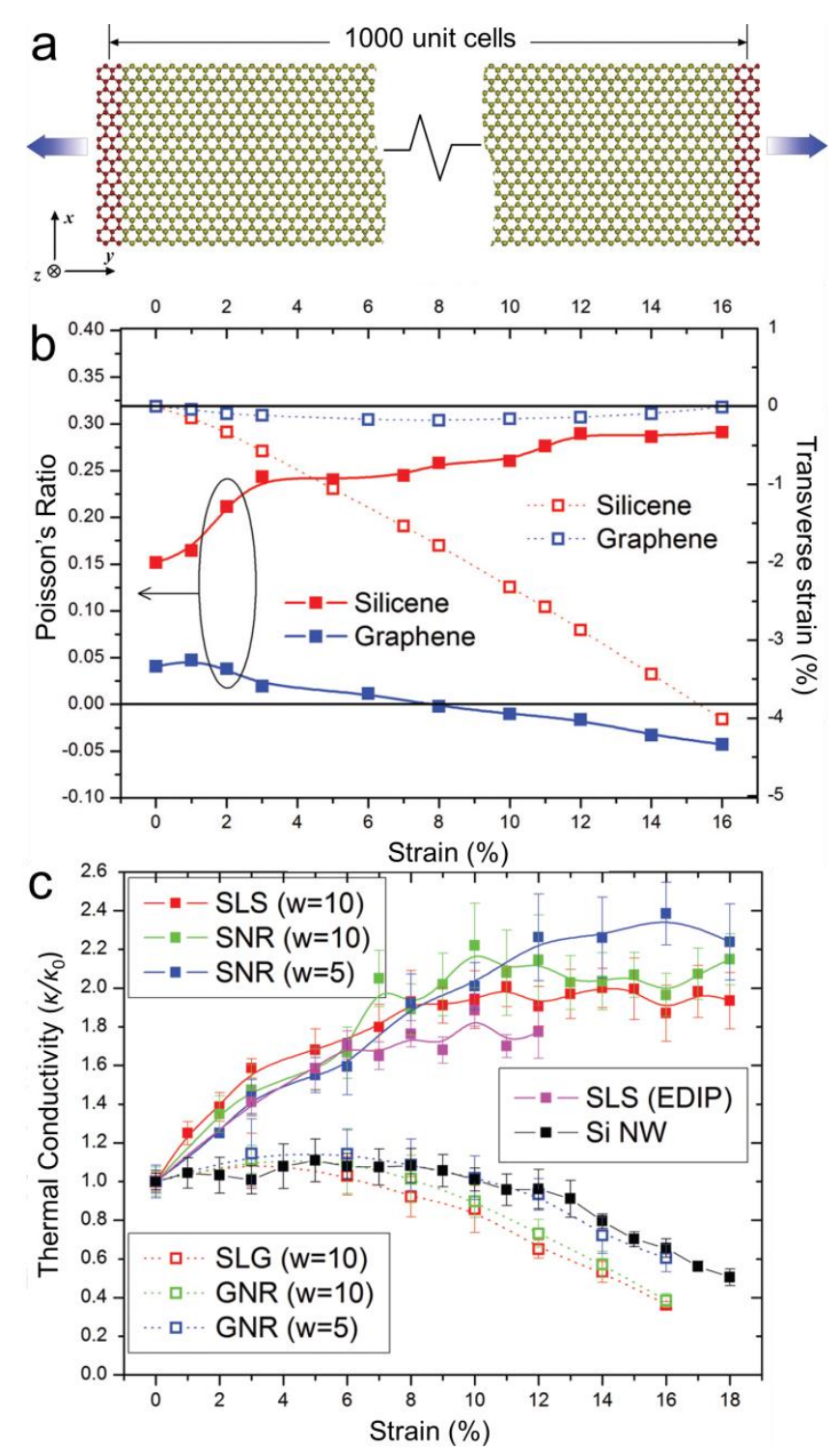

Figure 4. (a) zigzag silicene sheet used as model system in the MD simulations. The $x$ axis (transverse direction) is along the armchair edge; the $y$-axis (longitudinal direction) is along the zigzag edge, and the uniaxial tension is applied along the $y$-axis direction. (b) Comparison of Poisson's ratio (left axis) and transverse strain (right axis) as a function of uniaxial stretching strain between single-layer silicene and graphene sheet. (c) Comparison of the relative thermal conductivities $\left(\kappa^{\prime} / \kappa_{0}\right)$ where $\kappa_{0}$ is at $0 \%$ strain and $w$ indicating the width (number of unit cells). SLS and SLG: single layer silicene and graphene, SNR and GNR: silicene and graphene nanoribbon, Si NW: silicon nanowire. All silicene and graphene layers are zigzag and are 1000 and 1500 unit cells long, respectively. Reproduced with permission. ${ }^{[79]}$ Copyright 2013, American Physical Society.

Kaltsas et al. have studied the effect of axial strain and compression on silicane and germanane sheets, through theoretical simulations. ${ }^{[80]}$ The structures are predicted to ripple or undergo a transformation from chair- to washboard-like structure. Such aspects can be utilized for creating patterned superstructures of chair/washboard-like silicane and germanane that 
possess distinct properties. As free standing silicane and germanane sheets have already been realized, experimental studies confirming such exceptional mechanical properties are expected to follow rapidly. Experimental progress in this area is of utmost importance in establishing a novel pathway for mechanically induced tunability which will facilitate the development of functional nanoelectromechanical systems.

Phosphorene on the other hand, exhibits superior mechanical flexibility in comparison to other 2D materials (graphene and $\mathrm{MoS}_{2}$ ) with its Young's modulus one order of magnitude smaller. ${ }^{[81-83]}$ Unlike graphene $(\sim 1 \mathrm{TPa})$, the Young's modulus of phosphorene $(\sim 94 \mathrm{GPa})$ is found to be direction dependent (166 and $44 \mathrm{GPa}$ along zig-zag and armchair directions respectively). ${ }^{[81]}$ Theoretical studies show that phosphorene can withstand up to $30 \%$ axial strain, owing to the puckered nature of the crystal lattice. ${ }^{[81]}$ Such an effect is attributed to the tensile strain stretching the pucker in the crystal lattice rather than extending the phosphorus bond length. Such mechanical flexibility along with its excellent electronic capabilities, render phosphorene as a competitive alternative for modern flexible electronics.

\subsection{Thermal Properties}

The mechanical For Group IV elemental sheets, their thermal conductivity and the thermoelectric parameters have been theoretically studied. The total thermal conductivity $\kappa=$ $\kappa_{\mathrm{e}}+\kappa_{\mathrm{p}}$, where $\kappa_{\mathrm{e}}$ and $\kappa_{\mathrm{p}}$ are electron and phonon contributions, respectively. While $\kappa$ of graphene has been predicted to be in a high $\sim 10^{3} \mathrm{Wm}^{-1} \mathrm{~K}^{-1}$ range, experimental evidence has pointed to even higher values $\left(4.4\right.$ to $\left.5.8 \times 10^{3} \mathrm{Wm}^{-1} \mathrm{~K}^{-1}\right) \cdot{ }^{[84]}$ However, in comparison to graphene, silicene is predicted to have a significantly lower $\kappa$ at least by an order of magnitude. ${ }^{[79,84-85]}$ Through molecular dynamics simulations and anharmonic lattice dynamics calculations, Zhang et al. have shown that the longitudinal phonon modes dominate the thermal conductivity in silicene, in contrast to that of graphene. ${ }^{[84]}$ These studies attribute the low thermal conductivity in silicene to a relatively short phonon mean-free path. This implies that a larger number of scattering centers are available for acoustic phonons which result in 
the obvious lower thermal conductivity, which is desired for conventional thermoelectric applications. Studies on vibrational density of states also relate this phenomenon to less excitable phonons possessed by silicene in comparison to those of graphene. ${ }^{[86]}$

Through theoretical simulations, Hu et al. have illustrated the anomalous effect of strain on the thermal conductivity. ${ }^{[79]}$ Figure $4 \mathrm{c}$ presents the effect of strain on the thermal conductivity of silicene and graphene sheets for a range of crystal dimensions. Under strain, silicene shows an increase in $\kappa$ while graphene's $\kappa$ seemed to decrease. Such a behavior is attributed to the buckled nature of silicene allowing it to deform flexibly in the lateral direction reducing phonon scattering (leading to an increase in $\kappa$ ), whereas the flat planar nature of graphene under strain causes lattice anharmonicity, resulting in a decrease in $\kappa .^{[79]}$

The theoretical studies of thermal conductivity have also been extended to investigating the thermoelectric properties of these $2 \mathrm{D}$ materials. ${ }^{[87-89]}$ Thermoelectric effect is the conversion of a temperature gradient across a material into an electrical current and is caused by electronphonon drag through the material. The efficiency of this process is characterized by the thermoelectric figure of merit $Z T{ }^{[88]}$

$$
Z T=\frac{\sigma S^{2} T}{\kappa}
$$

where $\sigma$ is the electrical conductivity, $S$ is the Seebeck co-efficient and $T$ is the temperature. As described by Equation 1, for optimal ZT in conventional thermoelectric applications, a material should possess large $\sigma$ and $S$, while exhibiting low $\kappa$. Described by the WiedemannFranz law, engineering $\sigma$ and $\kappa$ to achieve optimal $Z T$ in a metal is less probable as they are proportionally related to each other. ${ }^{[88,90]}$ While a $Z T$ of 1 is considered to be competitive, values above 3 are desirable to compete against established energy generation technologies. ${ }^{[87]}$ Graphene has been proven to be inefficient as a thermoelectric material due to its high $\kappa$, and poor compatibility in being integrated with the current generation of silicon based technologies. ${ }^{[88-89]}$ On the other hand, silicene and germanene possess excellent 
electronic characteristics similar to that of graphene while exhibiting a poor $\kappa$ and excellent integrability with the well-established silicon technology. Although the ZT of intrinsic silicene is predicted to be $\sim 1{ }^{[88]}$ through theoretical analyses Pan et al. have shown an increase in $Z T$ of up to 2.8 via introducing appropriate $p$ - or $n$-type dopants, into the crystal lattice. ${ }^{[87]}$ Furthermore, the $S$ of pristine silicene sheets is calculated to be $\sim 80 \mu \mathrm{VK}^{-1}$ and multifold enhancements in $S$ are predicted from an opening energy gap are also reported. ${ }^{[91]}$

Phosphorene is predicted to exhibit highly anisotropic thermal and thermoelectric properties depending on the type and level of doping. ${ }^{[67,92]}$ First principle studies reveal that optimum $p$ type doping, can result in a room temperature $Z T$ of 0.3 and a thermoelectric power factor $(P F)$ of $138.9 \mu \mathrm{Wcm}^{-1} \mathrm{~K}^{-2}\left(S=197 \mu \mathrm{VK}^{-1}\right)$ mainly limited by the high lattice $\kappa \cdot{ }^{[92]}$ In contrast, black phosphorus is predicted to show optimum thermoelectric properties under $n$-type doping conditions $\left(Z T=0.22, P F=118.4 \mu \mathrm{Wcm}^{-1} \mathrm{~K}^{-2}\right)$. The lattice $\kappa$ can be manipulated depending on the application. For instance, thermopower wave sources benefit from a high $\kappa$, while conventional thermoelectric applications require a low $\kappa^{[93-96]}$ The thermal conductivity can be engineered by introducing additional scattering sites (intercalants) within the van der Waals gap, which can reduce $\kappa$ for conventional thermoelectric applications. Theoretical studies also reveal strain engineering as a viable alternative for enhancing the $Z T$ of black phosphorus. At optimal strain levels $(\sim 7 \%), Z T$ values are predicted to reach $0.521 .^{[67]}$ Such flexibility in engineering the thermal and thermoelectric properties is of key value, in the development of highly competitive, low dimensional thermoelectric systems.

\section{Realizing 2D Elemental Sheets}

2D elemental sheets can be achieved through bottom up synthesis techniques and top down exfoliation approaches. 


\subsection{Deposition Techniques}

Bottom-up synthesis methods cater for two distinct purposes: (1) Controlled growth of a single isolated 2D sheet and (2) synthesis of bulk, layered crystals suitable for separating individual 2D sheets through top-down approaches. Thus far, surface assisted epitaxial growth under ultra-high vacuum has been the only method to realize monolayers of elemental $2 \mathrm{D}$ sheets. Highly ordered growth assisting substrates such as $\mathrm{Ag}$, Ir, and $\mathrm{ZrBr}_{2}$ have proven successful in realizing silicene nano ribbons, and nanosheets. ${ }^{[11,32,36,97-103]}$ Typically, the substrates are cleaned with argon ion bombardment until a highly ordered (111) oriented surface is achieved. Subsequently, 2D silicene growth is achieved through direct evaporation of silicon with highly controlled deposition flux, at various substrates temperatures (400 $670 \mathrm{~K}) .{ }^{[11,32,36,97-101]}$ This technique can also be extended to the production of germanene, ${ }^{[104]}$ and stanene nanosheets. Figure 5 shows a typical scanning tunneling microscopic (STM) image of a buckled silicene layer grown on an Ag (111) surface and is in clear agreement with calculated lattice parameters. Detailed review on the deposition of silicene and graphene on various transition metal surfaces is available elsewhere. ${ }^{[105]}$

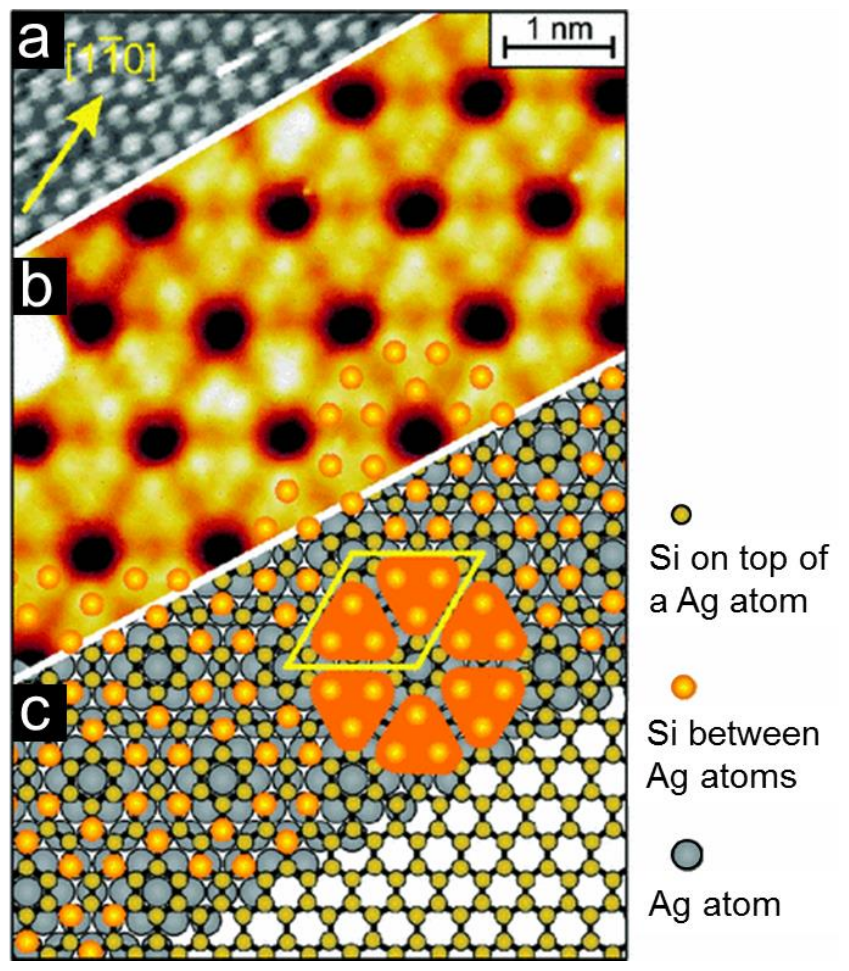


Figure 5. Silicene on Ag (111) surface: Filled state STM images of (a) Ag (111) surface and (b) silicene on Ag. (c) Schematic overlay describing the observed STM images in (a) and (b). Reproduced with permission. ${ }^{[11]}$ Copyright 2012, American Physical Society.

From first principle studies, Bhattacharya et al. have investigated various other ordered (111) substrates (AlAs, AlP, GaAs, GaP, $\mathrm{ZnS}$, and $\mathrm{ZnSe}$ ) for the epitaxial growth of silicene. ${ }^{[106]}$ These studies reveal interesting insights into the influence of substrates, affecting the doping characteristics in the 2D nanosheets. It is reported that metal and non-metal terminated elemental sheets behave like typical $n$ - and $p$-doped materials, respectively. ${ }^{[106]}$ Such substrate dependent doping conditions portray significant potential in facile bandgap engineering of such elemental sheets for various electronic applications. Theoretical studies also indicate GaS to be an ideal candidate for forming heterosheets with silicene. ${ }^{[107]}$ In such structures, silicene is predicted to have retained its desirable characteristics such as Dirac-like band structure and the stable low buckled nature, which are typically observed in its free standing state. $^{[107]}$

Chemical methods have also been employed for synthesizing layered crystals of hydrogen decorated poly-silane and germanane $(\mathrm{SiH}$ and $\mathrm{GeH})$. Low temperature topochemical deintercalation of calcium silicide and germanide $\left(\mathrm{CaSi}_{2}\right.$ and $\left.\mathrm{CaGe}_{2}\right)$ in aqueous $\mathrm{HCl}$, has been shown to produce large layered crystals of $\mathrm{SiH},{ }^{[108-109]}$ and $\mathrm{GeH} .{ }^{[23,110]}$ On the other hand, black phosphorus crystals are typically obtained through high temperature treatment $\left(\sim 1000{ }^{\circ} \mathrm{C}\right)$ of red phosphorus (relatively unstable polymorph of phosphorus), under high pressure. ${ }^{[12]}$ Such reactions under catalyzed conditions have also been demonstrated to produce crystalline growth of black phosphorus under slightly low temperature conditions. ${ }^{[20]}$ A similar time efficient technique of low pressure transport method has also been demonstrated to produce high purity large area black phosphorus crystals. ${ }^{[111]}$ However, bottom-up phosphorene synthesis approaches are yet to be explored. 


\subsection{Exfoliation techniques}

Top-down exfoliation methods involve, extracting individual sheets from their growth assisting substrates or their layered bulk crystals via various techniques. In the case of such elemental materials, chemical techniques have been employed for isolating 2D sheets off their growth substrates. Standard mechanical and liquid cleaving techniques have been explored to extract 2D sheets from their bulk layered crystals. However, mechanical and liquid exfoliation techniques can only be applied to layered crystals that are composed of 2D planar sheets, which are held together by weak van der Waals forces. Hence, they are inapplicable for producing silicene, gemanene and stanene sheets, as they have not been known to exist in a layered bulk form. Novel plasma $\left(\mathrm{Ar}^{+}\right)$assisted thinning technique has been recently demonstrated for producing monolayers of phosphorene, a similar alternative to laser assisted thinning of $\mathrm{MoS}_{2 .}{ }^{[112]}$ The feasibility of such techniques on phosphorene, enables prospective chemical and other high energy thinning alternatives.

\subsubsection{Chemical exfoliation}

The atomic As elemental sheets are mainly grown on substrates (so far), inserting intercalants between the $2 \mathrm{D}$ sheets and their growth substrate opens a viable pathway to realise free standing elemental sheets. Tsetseris et al. have used first principle studies to investigate the interactions of $\mathrm{H}, \mathrm{Li}$ and $\mathrm{Ca}$ atoms with silicene. ${ }^{[24,113]}$ The calculations show that adequate levels of $\mathrm{H}$ insertion, it forms a freestanding decorated silicane structure in separating the silicene sheet from its growth substrate. This process has been experimentally demonstrated in the case of extracting silicene nano ribbons from Ag substrates and is yet to be extended to sheets. ${ }^{[114-115]} \mathrm{Li}$ was observed to have a preferential state of bonding on top of silicene proving inadequate for exfoliation purposes. ${ }^{[113]}$ However, Ca was predicted to intercalate in between the interface and detach the $2 \mathrm{D}$ layers, resulting in a metallic silicene structure. ${ }^{[24]}$ Kaloni et al. have predicted the hydrogenation of germanene on GaAs substrate, resulting in free standing, germanene sheets with an inherent energy gap $(24 \mathrm{meV})$. This is a consequence 
of the hydrogen atoms intercalating and bonding with the substrate surface, instead of the germanene sheet. ${ }^{[16]}$ The aforementioned studies reveal the nature of various adatoms, each favoring a discrete range of interactions with the elemental sheets, depending on their density of electronic states. Such aspects can be utilized for realizing and engineering 2D elemental sheets that cater for distinct applications.

\subsubsection{Mechanical Exfoliation}

Mechanical exfoliation technique is known to produce the most pristine 2D sheets in the case of graphene and other $2 \mathrm{D}$ materials. ${ }^{[1,3,117]}$ This process involves the separation of individual 2D sheets through the application of mechanical force, commonly applied using adhesive tape. As discussed earlier, decorated elemental structures exist in a layered crystal form. Bianco et al. have used mechanical exfoliation to isolate and transfer mono-layered $\mathrm{GeH}$ sheets onto $\mathrm{SiO}_{2} / \mathrm{Si}$ substrates (Figure 6a). ${ }^{[23]}$ Such sheets have shown relatively long term resistance to oxidation and high thermal stability. ${ }^{[23]}$ This report opens a potential complementary route for realizing $\mathrm{SiH}$ monolayers. As discussed in Section 3.1, large layered $\mathrm{SiH}$ crystals have been successfully synthesized and similar to $\mathrm{GeH}$, such crystals may be subjected to mechanical exfoliation. Such a technique would provide a pathway to experimentally study the potential properties of silicene's decorated structures and assess their viability in electronic applications. Similarly, phosphorene has been attained on $\mathrm{SiO}_{2} / \mathrm{Si}$ substrates via the mechanical exfoliation of black phosphorous crystals. ${ }^{[12-13]}$ Figure $6 \mathrm{~b}$ illustrates the AFM image of a mono-layer phosphorene sheet (inset: optical image of bulk black phosphorous crystals) with a thickness of $\sim 0.85 \mathrm{~nm}$, closely corresponding to its mono-layer height. ${ }^{[13]}$ 


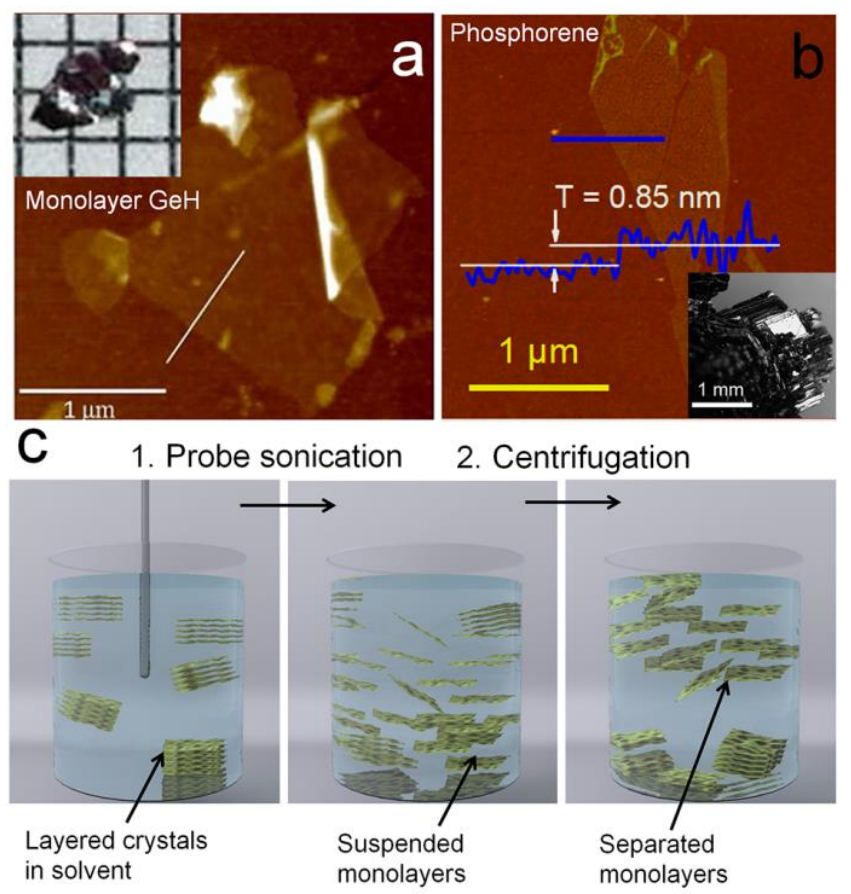

Figure 6. AFM images of mono-layer (a) $\mathrm{GeH}$ and (b) phosphorene sheets with insets indicating their bulk crystals respectively (c) Artist's illustration of the liquid exfoliation technique. Reproduced with permission. (a), ${ }^{[23]}$ (b), ${ }^{[13,20]}$, and (b) inset. ${ }^{[20]}$ Copyright 20072014, American Chemical Society.

\subsubsection{Liquid Exfoliation}

Liquid cleaving techniques involve the exfoliation of monolayers through ultra-sonication of suspended layered crystals. Subsequently, the exfoliated suspension is centrifuged to separate the lighter monolayers from the bulk counterparts. Figure $6 \mathrm{c}$ illustrates the process of a typical liquid phase exfoliation method. Although this approach is a higher yield process for producing $2 \mathrm{D}$ sheets compared mechanical exfoliation, it suffers in the aspect of purity. Liquid exfoliation techniques have not yet been adopted in the production decorated elemental sheets, with the exception of graphene. The reactivity of the decorated elemental sheets with solvents may play a negative role in the application of liquid exfoliation processes. With suitable solvents (that do not react with the material), black phosphorous can be subjected to liquid exfoliation techniques in order to produce large area phosphorene sheets, however it is yet to be demonstrated. 


\section{Conclusions and Future Outlook}

Due to their promising properties, elemental graphene analogues can be eventually adopted for a wide range of applications. Elemental silicene and germanene can be considered as an excellent alternative avenue for overcoming the technological barriers that have hampered the current silicon and germaninum based electronics. Such a two dimensional polymorph of silicon and germanium has the potential to extend the Moore's law, by overcoming the dimensional constraints of the current generation transistors. Furthermore, it can be expected that the ballistic transport properties observed in the elemental sheets would also allow the possibilities of realizing ultra-high speed nanotransistors. Silicene and germanene, can also be easily integrated with existing electronic technologies while graphene and other 2D materials require significant modifications to the established technological framework. In addition, the inherent Dirac fermions and the predicted quantum spin-hall effects at accessible temperatures, render silicene and germanene as feasible candidates for spintronics. Their robustness and high resistance to oxidation are certainly added advantages and portray render them desirable for durable device technology. Such potential can also be extrapolated to stanene, which demands further investigations.

Phosphorene on the other hand, stands besides graphene when it comes to integration with current technologies. However, unlike graphene, it is a $2 \mathrm{D}$ semiconductor with a direct bandgap which is a desired feature. Recent advancements made in phosphorene based electronics reveal its outstanding capabilities compared to that of any other 2D semiconductor and should inspire a rapid exploration of phosphorene based high speed electronics. Due to its narrow bandgap nature and facile tunability, phosphorene can very well be utilized to cater for various optoelectronic, battery electrode, sensor and nano-optics applications. Many of 2D phosphorene's electronic, mechanical, optical and thermal properties are yet to be experimentally characterized and further studies may project its maximum potential. 
Although less than par with graphene, the mechanical properties of other Group IV elemental sheets have shown anomalous behavior in comparison. Silicene and germanene show excellent flexibility and compressibility than graphene, while strain engineering has been envisioned to modify their electronic and thermal properties. Such aspects make these elemental sheets plausible candidates for establishing nano electromechanical systems and sensors that can be well integrated with the current generation of device technology. The decoupling of the key thermoelectric parameters is envisaged to provide precise control to isolatively engineer their thermoelectric behaviors. Such unique capabilities of silicene along with its ballistic transport properties offer a promising potential for developing highly efficient thermoelectric applications. Such aspects can be extrapolated to germanene and stanene and are yet to be shown.

Tunability of the material properties in such elemental sheets offers a fascinating prospect of engineering discrete applications. Various techniques such as doping/decorating, strain engineering and application of an external field have been theoretically shown to allow precise tunability of the fundamental properties. Specifically, decorated 2D semiconducting sheets of free standing silicane and germanane have already been achieved and their electronic properties were observed to differ substantially from intrinsic semi-metallic elemental sheets. It is highlighted that further experimental investigations should be pursued, in order to exploit the quantum dynamics that render such materials susceptible to manipulations at a molecular level. Such unique features are expected to overcome fundamental constraints that have imposed dimensional physical limits on the evolution of faster, smaller and efficient nanoelectronics. Furthermore, studies on decorated structures reveal the excellent energy and charge storage capabilities of Group IV elemental sheets. ${ }^{[113,}$ 118-119] This aspect can open a new horizon for low dimensional, competitive and durable storage technologies. 
The lack of progress in facile synthesis techniques has limited the experimental advances to support most of the theoretical predictions. So far, the available synthesis approaches in producing graphene analogous Group IV elemental materials, have been limited to substrate assisted epitaxial growth. Such a limitation is expected due to the metastable nature of these elemental sheets. Alternative approaches to produce such $2 \mathrm{D}$ sheets and cleaving them off their growth substrates are yet to be explored. Phosphorene on the other hand, is a stable, free standing 2D material and black phosphorus has already been realized using chemical transport techniques. However, facile bottom-up growth techniques for realizing monolayer phosphorene is yet to be demonstrated in order to realize the true potential of phosphorene. As such, advances in synthesis techniques for producing graphene analogous $2 \mathrm{D}$ elemental sheets will be a significant milestone towards harnessing their tremendous potential for the development of next generation of electronic, optoelectronic and spintronic applications. 


\section{Acknowledgements}

The authors acknowledge support from the Australian Research Council (ARC) with project support through Discovery Projects DP130100062 (S.S.) and DP140100170 (M.B.) and Australian Post-Doctoral Fellowships through Discovery Projects DP1092717 (M.B.) and DP110100262 (S.S.).

Received: ((will be filled in by the editorial staff))

Revised: ((will be filled in by the editorial staff))

Published online: ((will be filled in by the editorial staff $)$ ) 
[1] K. S. Novoselov, A. K. Geim, S. V. Morozov, D. Jiang, Y. Zhang, S. V. Dubonos, I. V. Grigorieva, A. A. Firsov, 2004, 306, 666.

[2] K. S. Novoselov, A. K. Geim, S. V. Morozov, D. Jiang, M. I. Katsnelson, I. V. Grigorieva, S. V. Dubonos, A. A. Firsov, Nature 2005, 438, 197.

[3] S. Balendhran, S. Walia, H. Nili, J. Z. Ou, S. Zhuiykov, R. B. Kaner, S. Sriram, M. Bhaskaran, K. Kalantar-zadeh, Adv. Funct. Mater. 2013, 23, 3952.

[4] Q. H. Wang, K. Kalantar-zadeh, A. Kis, J. N. Coleman, M. S. Strano, Nat. Nanotechnol. 2012, 7, 699.

[5] Q. Tang, Z. Zhou, Prog. Mater. Sci. 2013, 58, 1244.

[6] M. Chhowalla, H. S. Shin, G. Eda, L. J. Li, K. P. Loh, H. Zhang, Nat. Chem. 2013, 5, 263.

[7] L. C. L. Yan Voon, G. G. Guzmán-Verri, MRS Bull. 2014, 39, 366.

[8] S. Balendhran, J. Deng, J. Z. Ou, S. Walia, J. Scott, J. Tang, K. L. Wang, M. R. Field, S. Russo, S. Zhuiykov, M. S. Strano, N. Medhekar, S. Sriram, M. Bhaskaran, K. Kalantar-zadeh, Adv. Mater. 2013, 25, 109.

[9] S. Balendhran, S. Walia, M. Alsaif, E. P. Nguyen, J. Z. Ou, S. Zhuiykov, S. Sriram, M. Bhaskaran, K. Kalantar-zadeh, ACS Nano 2013, 7, 9753.

[10] S. Walia, S. Balendhran, Y. Wang, R. Ab Kadir, A. Sabirin Zoolfakar, P. Atkin, J. Zhen Ou, S. Sriram, K. Kalantar-zadeh, M. Bhaskaran, Appl. Phys. Lett. 2013, 103, 232105.

[11] P. Vogt, P. De Padova, C. Quaresima, J. Avila, E. Frantzeskakis, M. C. Asensio, A. Resta, B. Ealet, G. Le Lay, Phys. Rev. Lett. 2012, 108, 155501.

[12] L. Li, Y. Yu, G. J. Ye, Q. Ge, X. Ou, H. Wu, D. Feng, X. H. Chen, Y. Zhang, Nat. Nanotechnol. 2014, 9, 372.

[13] H. Liu, A. T. Neal, Z. Zhu, Z. Luo, X. Xu, D. Tománek, P. D. Ye, ACS Nano 2014, 8, 4033.

[14] S. Z. Butler, S. M. Hollen, L. Y. Cao, Y. Cui, J. A. Gupta, H. R. Gutierrez, T. F. Heinz, S. S. Hong, J. X. Huang, A. F. Ismach, E. Johnston-Halperin, M. Kuno, V. V.

Plashnitsa, R. D. Robinson, R. S. Ruoff, S. Salahuddin, J. Shan, L. Shi, M. G. Spencer, M. Terrones, W. Windl, J. E. Goldberger, ACS Nano 2013, 7, 2898.

[15] A. Kara, H. Enriquez, A. P. Seitsonen, L. C. Lew Yan Voon, S. Vizzini, B. Aufray, H. Oughaddou, Surf. Sci. Rep. 2012, 67, 1.

[16] Y. Ding, Y. L. Wang, J. Phys. Chem. C 2013, 117, 18266.

[17] Y. Xu, B. Yan, H.-J. Zhang, J. Wang, G. Xu, P. Tang, W. Duan, S.-C. Zhang, Phys. Rev. Lett. 2013, 111, 136804.

[18] L. Matthes, O. Pulci, F. Bechstedt, J. Phys.-Condes. Matter 2013, 25, 395305.

[19] A. Manjanath, V. Kumar, A. K. Singh, Phys. Chem. Chem. Phys. 2014, 16, 1667.

[20] S. Lange, P. Schmidt, T. Nilges, Inorg. Chem. 2007, 46, 4028.

[21] J. C. Garcia, D. B. de Lima, L. V. C. Assali, J. o. F. Justo, J. Phys Chem. C 2011, 115, 13242.

[22] S. Trivedi, A. Srivastava, R. Kurchania, J. Comput. Theor. Nanosci. 2014, 11, 781.

[23] E. Bianco, S. Butler, S. Jiang, O. D. Restrepo, W. Windl, J. E. Goldberger, ACS Nano 2013, 7, 4414.

[24] L. Tsetseris, D. Kaltsas, Chem. Phys. Phys. Chem. 2014, 16, 5183.

[25] H. Sahin, F. M. Peeters, Phys. Rev. B 2013, 87, 085423.

[26] L. C. Lew Yan Voon, E. Sandberg, R. S. Aga, A. A. Farajian, Appl. Phys. Lett. 2010, 97, 163114.

[27] S. Lebègue, M. Klintenberg, O. Eriksson, M. I. Katsnelson, Phys. Rev. B 2009, 79, 245117.

[28] M. Houssa, E. Scalise, K. Sankaran, G. Pourtois, V. V. Afanas'ev, A. Stesmans, Appl. Phys. Lett. 2011, 98, 223107. 
[29] M. Liu, Y. Han, L. Tang, J.-F. Jia, Q.-K. Xue, F. Liu, Phys. Rev. B 2012, 86, 125427.

[30] M. Houssa, G. Pourtois, V. V. Afanas'ev, A. Stesmans, Appl. Phys. Lett. 2010, 96, 082111.

[31] S. Lebegue, O. Eriksson, Phys. Rev. B 2009, 79, 115409.

[32] L. Chen, C.-C. Liu, B. Feng, X. He, P. Cheng, Z. Ding, S. Meng, Y. Yao, K. Wu, Phys. Rev. Lett. 2012, 109, 056804.

[33] C.-C. Liu, H. Jiang, Y. Yao, Phys. Rev. B 2011, 84, 195430.

[34] P. De Padova, P. Vogt, A. Resta, J. Avila, I. Razado-Colambo, C. Quaresima, C. Ottaviani, B. Olivieri, T. Bruhn, T. Hirahara, T. Shirai, S. Hasegawa, M. C. Asensio, G. Le Lay, Appl. Phys. Lett. 2013, 102, 163106.

[35] R. Arafune, C. L. Lin, R. Nagao, M. Kawai, N. Takagi, Phys. Rev. Lett. 2013, 110, 229701.

[36] C.-L. Lin, R. Arafune, K. Kawahara, M. Kanno, N. Tsukahara, E. Minamitani, Y. Kim, M. Kawai, N. Takagi, Phys. Rev. Lett. 2013, 110, 076801.

[37] C. L. Kane, E. J. Mele, Phys. Rev. Lett. 2005, 95, 226801.

[38] C.-C. Liu, W. Feng, Y. Yao, Phys. Rev. Lett. 2011, 107, 076802.

[39] B. A. Bernevig, T. L. Hughes, S.-C. Zhang, Science 2006, 314, 1757.

[40] M. König, S. Wiedmann, C. Brüne, A. Roth, H. Buhmann, L. W. Molenkamp, X.-L. Qi, S.-C. Zhang, Science 2007, 318, 766.

[41] Y. Ma, Y. Dai, M. Guo, C. Niu, B. Huang, J. Phys. Chem. C 2012, 116, 12977.

[42] Y. Cai, C.-P. Chuu, C. M. Wei, M. Y. Chou, Phys. Rev. B 2013, 88, 245408.

[43] H. Liu, J. Gao, J. Zhao, J. Phys. Chem. C 2013, 117, 10353.

[44] N. D. Drummond, V. Zólyomi, V. I. Fal'ko, Phys. Rev. B 2012, 85, 075423.

[45] H. H. Gurel, V. O. Ozcelik, S. Ciraci, J. Phys.-Condes. Matter 2013, 25, 305007.

[46] Z. Y. Ni, Q. H. Liu, K. C. Tang, J. X. Zheng, J. Zhou, R. Qin, Z. X. Gao, D. P. Yu, J. Lu, Nano Lett. 2012, 12, 113.

[47] Y. Liang, V. Wang, H. Mizuseki, Y. Kawazoe, J. Phys.-Condes. Matter 2012, 24.

[48] Y. Ding, Y. Wang, Appl. Phys. Lett. 2012, 100, 083102.

[49] T. H. Osborn, A. A. Farajian, O. V. Pupysheva, R. S. Aga, L. C. Lew Yan Voon, Chem. Phys. Lett. 2011, 511, 101.

[50] R. Quhe, R. Fei, Q. Liu, J. Zheng, H. Li, C. Xu, Z. Ni, Y. Wang, D. Yu, Z. Gao, J. Lu, Scientific Rep. 2012, 2, 853.

[51] Y. L. Wang, Y. Ding, Phys. Status Solidi-Rapid Res. Lett. 2013, 7, 410.

[52] P. De Padova, C. Quaresima, B. Olivieri, P. Perfetti, G. Le Lay, J. Phys. D-Appl. Phys. 2011, 44, 312001.

[53] Y. Ding, Y. Wang, J. Phy. Chem. C 2014, 118, 4509.

[54] M. Nagao, A. Hayashi, M. Tatsumisago, J. Power Sources 2011, 196.

[55] J. Sun, G. Zheng, H.-W. Lee, N. Liu, H. Wang, H. Yao, W. Yang, Y. Cui, Nano Lett. 2014, 14, 4573.

[56] L.-Q. Sun, M.-J. Li, K. Sun, S.-H. Yu, R.-S. Wang, H.-M. Xie, J. Phys. Chem. C 2012, $116,14772$.

[57] S. Zhang, J. Yang, R. Xu, F. Wang, W. Li, M. Ghufran, Y.-W. Zhang, Z. Yu, G. Zhang, Q. Qin, Y. Lu, ACS Nano 2014, DOI: 10.1021/nn503893j.

[58] V. Tran, R. Soklaski, Y. Liang, L. Yang, Phys. Rev. B 2014, 89, 235319.

[59] Y. Du, C. Ouyang, S. Shi, M. Lei, J. Appl. Phys. 2010, 107, 093718.

[60] A. N. Rudenko, M. I. Katsnelson, Phys. Rev. B 2014, 89, 201408.

[61] S. Das, W. Zhang, M. Demarteau, A. Hoffmann, M. Dubey, A. Roelofs, Nano Lett. 2014, DOI: $10.1021 / \mathrm{nl} 5025535$.

[62] A. Ramasubramaniam, A. R. Muniz, Phys. Rev. B 2014, 90, 085424.

[63] R. Fei, L. Yang, Nano Lett. 2014, 14, 2884. 
[64] X. Han, H. Morgan Stewart, S. A. Shevlin, C. R. A. Catlow, Z. X. Guo, Nano Lett. 2014, 14, 4607.

[65] X. Peng, Q. Wei, A. Copple, Phys. Rev. B 2014, 90, 085402.

[66] A. S. Rodin, A. Carvalho, A. H. Castro Neto, Phys. Rev. Lett. 2014, 112, 176801.

[67] G. Qin, Z. Qin, S.-Y. Yue, H.-J. Cui, Q.-R. Zheng, Q.-B. Yan, G. Su, arXiv preprint 2014, arXiv:1406.0261.

[68] S. P. Koenig, R. A. Doganov, H. Schmidt, A. H. Castro Neto, B. Özyilmaz, Appl. Phys. Lett. 2014, 104, 103106.

[69] K. Lam, Z. Dong, J. Guo, IEEE Electron Device Lett. 2014, 35, 963.

[70] H. Liu, A. T. Neal, M. Si, Y. Du, P. D. Ye, IEEE Electron Device Lett. 2014, 35, 795.

[71] J. Qiao, X. Kong, Z.-X. Hu, F. Yang, W. Ji, Nat. Commun. 2014, 5, 4475.

[72] M. Buscema, D. J. Groenendijk, S. I. Blanter, G. A. Steele, H. S. J. van der Zant, A. Castellanos-Gomez, Nano Lett. 2014, 14, 3347.

[73] T. Hong, B. Chamlagain, W. Lin, H.-J. Chuang, M. Pan, Z. Zhou, Y.-Q. Xu, Nanoscale 2014, 6, 8978.

[74] M. Buscema, D. J. Groenendijk, G. A. Steele, H. S. J. van der Zant, A. CastellanosGomez, Nat. Commun. 2014, 5, 4651.

[75] J. Dai, X. C. Zeng, J. Phys. Chem. Lett. 2014, 5, 1289.

[76] Y. Deng, Z. Luo, N. J. Conrad, H. Liu, Y. Gong, S. Najmaei, P. M. Ajayan, J. Lou, X. $\mathrm{Xu}, \mathrm{P}$. D. Ye, ACS Nano 2014, 8, 8292.

[77] L. Kou, T. Frauenheim, C. Chen, J. Phys. Chem. Lett. 2014, 5, 2675.

[78] Y. Wang, Y. Ding, Solid State Commun. 2013, 155, 6.

[79] M. Hu, X. Zhang, D. Poulikakos, Phys. Rev. B 2013, 87, 195417.

[80] D. Kaltsas, T. Tsatsoulis, O. G. Ziogos, L. Tsetseris, J. Chem. Phys. 2013, 139, 124709.

[81] Q. Wei, X. Peng, Appl. Phys. Lett. 2014, 104, 251915.

[82] S. Appalakondaiah, G. Vaitheeswaran, S. Lebègue, N. E. Christensen, A. Svane, Phys. Rev. B 2012, 86, 035105.

[83] J. Jin-Wu, S. P. Harold, J. Phys. D-Appl. Phys. 2014, 47, 385304.

[84] X. L. Zhang, H. Xie, M. Hu, H. Bao, S. Y. Yue, G. Z. Qin, G. Su, Phys. Rev. B 2014, 89, 054310.

[85] Q.-X. Pei, Y.-W. Zhang, Z.-D. Sha, V. B. Shenoy, J. Appl. Phys. 2013, 114, 033526.

[86] T. Y. Ng, J. J. Yeo, Z. S. Liu, Int. J. Mech. Mater. Des. 2013, 9, 105.

[87] L. Pan, H. J. Liu, X. J. Tan, H. Y. Lv, J. Shi, X. F. Tang, G. Zheng, Chem. Phys. Phy. Chem. 2012, 14, 13588.

[88] K. Yang, S. Cahangirov, A. Cantarero, A. Rubio, R. D'Agosta, Phys. Rev. B 2014, 89, 125403.

[89] K. Zberecki, M. Wierzbicki, J. Barnas, R. Swirkowicz, Phys. Rev. B 2013, 88, 115404.

[90] S. Walia, S. Balendhran, H. Nili, S. Zhuiykov, G. Rosengarten, Q. H. Wang, M. Bhaskaran, S. Sriram, M. S. Strano, K. Kalantar-zadeh, Prog. Mater. Sci. 2013, 58, 1443.

[91] Y. H. Yan, H. F. Wu, F. Jiang, H. Zhao, Eur. Phys. J. B 2013, 86, 457.

[92] H. Lv, W. Lu, D. Shao, Y. Sun, arXiv preprint 2014, arXiv:1404.5171.

[93] S. Walia, S. Balendhran, P. Yi, D. Yao, S. Zhuiykov, M. Pannirselvam, R. Weber, M. S. Strano, M. Bhaskaran, S. Sriram, K. Kalantar-zadeh, J. Phys. Chem. C 2013, 117, 9137.

[94] S. Walia, R. Weber, S. Balendhran, D. Yao, J. T. Abrahamson, S. Zhuiykov, M. Bhaskaran, S. Sriram, M. S. Strano, K. Kalantar-zadeh, Chem. Commun. 2012, 48, 7462.

[95] S. Walia, R. Weber, K. Latham, P. Petersen, J. T. Abrahamson, M. S. Strano, K. Kalantar-zadeh, Adv. Funct. Mater. 2011, 21, 2072. 
[96] S. Walia, R. Weber, S. Sriram, M. Bhaskaran, K. Latham, S. Zhuiykov, K. Kalantarzadeh, Energ. Environ. Sci. 2011, 4, 3558.

[97] L. Chen, H. Li, B. Feng, Z. Ding, J. Qiu, P. Cheng, K. Wu, S. Meng, Phys. Rev. Lett. 2013, 110, 085504.

[98] A. Fleurence, R. Friedlein, T. Ozaki, H. Kawai, Y. Wang, Y. Yamada-Takamura, Phys. Rev. Lett. 2012, 108, 245501.

[99] B. Lalmi, H. Oughaddou, H. Enriquez, A. Kara, S. Vizzini, B. Ealet, B. Aufray, Appl. Phy. Lett. 2010, 97, 223109.

[100] Z. Majzik, M. R. Tchalala, M. Svec, P. Hapala, H. Enriquez, A. Kara, A. J. Mayne, G. Dujardin, P. Jelinek, H. Oughaddou, J. Phys.-Condes. Matter 2013, 25, 225301.

[101] L. Meng, Y. Wang, L. Zhang, S. Du, R. Wu, L. Li, Y. Zhang, G. Li, H. Zhou, W. A. Hofer, H.-J. Gao, Nano Lett. 2013, 13, 685.

[102] C.-L. Lin, R. Arafune, K. Kawahara, N. Tsukahara, E. Minamitani, Y. Kim, N. Takagi, M. Kawai, Appl. Phys. Express 2012, 5, 045802.

[103] R. Arafune, C.-L. Lin, K. Kawahara, N. Tsukahara, E. Minamitani, Y. Kim, N. Takagi, M. Kawai, Surf. Sci. 2013, 608, 297.

[104] L. Li, S.-z. Lu, J. Pan, Z. Qin, Y.-q. Wang, Y. Wang, G.-y. Cao, S. Du, H.-J. Gao, Adv. Mater. 2014, DOI: 10.1002/adma.201400909.

[105] Q. Peng, J. Crean, A. K. Dearden, C. Huang, X. Wen, S. P. A. Bordas, S. De, Modern Phys. Lett. B 2013, 27, 1330017.

[106] A. Bhattacharya, S. Bhattacharya, G. P. Das, Appl. Phys. Lett. 2013, 103, 123113.

[107] Y. Ding, Y. Wang, Appl. Phy. Lett. 2013, 103, 043114.

[108] J. R. Dahn, B. M. Way, E. Fuller, J. S. Tse, Phys. Rev. B 1993, 48, 17872.

[109] J. He, J. S. Tse, D. D. Klug, K. F. Preston, J. Mater. Chem. 1998, 8, 705.

[110] G. Vogg, M. S. Brandt, M. Stutzmann, Adv. Mater. 2000, 12, 1278.

[111] T. Nilges, M. Kersting, T. Pfeifer, J. Solid State Chem. 2008, 181, 1707.

[112] W. Lu, H. Nan, J. Hong, Y. Chen, C. Zhu, Z. Liang, X. Ma, Z. Ni, C. Jin, Z. Zhang, Nano Res. 2014, 7, 853.

[113] G. A. Tritsaris, E. Kaxiras, S. Meng, E. Wang, Nano Lett. 2013, 13, 2258.

[114] M. E. Davila, A. Marele, P. De Padova, I. Montero, F. Hennies, A. Pietzsch, M. N. Shariati, J. M. Gomez-Rodriguez, G. Le Lay, Nanotechnol. 2012, 23, 385703.

[115] E. Salomon, T. Angot, Sci. Adv. Mater. 2011, 3, 354.

[116] T. P. Kaloni, U. Schwingenschlogl, J. Appl. Phys. 2013, 114, 184307.

[117] K. S. Novoselov, D. Jiang, F. Schedin, T. J. Booth, V. V. Khotkevich, S. V. Morozov, A. K. Geim, Proc. Natl. Acad. Sci. U. S. A. 2005, 102, 10451.

[118] T. Hussain, S. Chakraborty, R. Ahuja, Chemphyschem 2013, 14, 3463.

[119] T. Hussain, T. Kaewmaraya, S. Chakraborty, R. Ahuja, Phys. Chem. Chem. Phys. 2013, 15, 18900. 
A wide range of two-dimensional (2D) materials, are gaining increased attention owing to their unique properties that can be exploited for a plethora of applications. This article reviews the class of 2D elemental materials - silicene, germanene, stanene, and phosphorene - with emphasis on fundamental properties, synthesis techniques and their future prospects.

S. Balendhran,* S. Walia, H. Nili, S. Sriram, and M. Bhaskaran.*

\section{Elemental Analogues of Graphene: Silicene, Germanene, Stanene and Phosphorene}

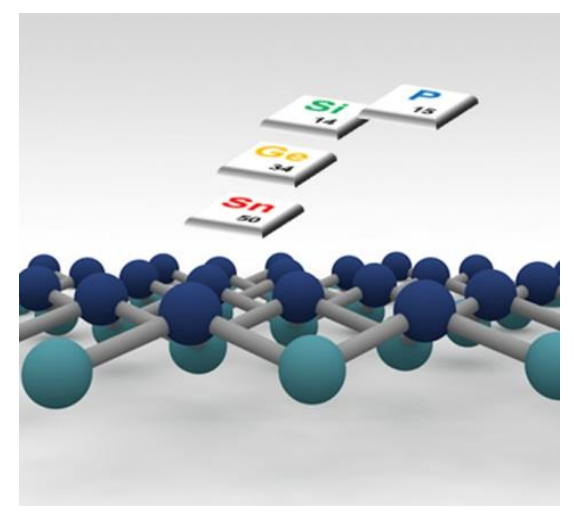

Sivacarendran Balendhran is a Post-doctoral Research Fellow at the School of Electrical and Computer Engineering, RMIT University, Australia. He received his Ph.D (2013) and B. Eng. Honours First Class (2009) from RMIT University. Sivacarendran's research interests include two-dimensional materials, nanomaterial synthesis, nanoelectronic devices and sensing systems.

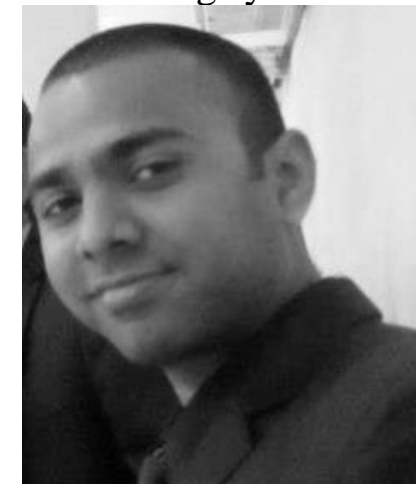


